\title{
Pueblos indígenas y la violencia social post Acuerdos de Paz en El Salvador
}

\author{
Indigenous Peoples and social violence \\ after the Peace Accords in El Salvador
}

\author{
Carlos Felipe Osegueda \\ Miguel Ángel Hernández² \\ Julissa Beatriz Beltrán-Hernández ${ }^{3}$ \\ Mariela Abigail López-Morales ${ }^{4}$ \\ Wendy Marisol Parada-Mónico ${ }^{5}$ \\ Katherine Yaneth Ponce-Juárez ${ }^{6}$ \\ Manuel Alexander Rodríguez-Machado ${ }^{7}$ \\ Raquel Aracely Rosales-Hernández ${ }^{8}$ \\ Vanessa Carolina Vásquez-Alvarado? \\ Universidad Tecnológica de El Salvador \\ carlos.osegueda@utec.edu.sv
}

\section{Resumen}

La población indígena en El Salvador ha experimentado diferentes formas de violencia, discriminación, sometimiento e imposición desde la Colonia como sistema económico adverso a sus cosmovisiones. Dicho escenario ha generado consecuencias adversas en la tenencia de tierra, exclusión y marginación social hacia el indígena, lo cual ha permeado en el imaginario colectivo de las nuevas generaciones, negación que se refleja en su vestimenta y habla. En ese sentido, la finalidad del presente artículo es estudiar el fenómeno de la violencia social hacia los

\section{Abstract}

The indigenous population in El Salvador has gone through different forms of violence, discrimination, subjugation and imposition since Colonial times as an economic system that was adverse to their worldview. This scenario has generated adverse consequences to land ownership, exclusion and social marginalization towards the indigenous peoples; this situation has influenced the cosmovision of their new generations; this situation can be evidenced in their denial to wear their clothing and speak their tongue. In this sense, the goal

\footnotetext{
${ }^{1}$ Profesor titular de la asignatura de Realidad Nacional

${ }^{2}$ Estudiante investigador e instructor de la asignatura de Realidad Nacional

${ }^{3}$ Estudiante de la Licenciatura en Administración de Empresas

${ }^{4}$ Estudiante de la Licenciatura en Contaduría Pública

${ }^{5}$ Estudiante de la Licenciatura en Comunicaciones

${ }^{6}$ Estudiante de la Licenciatura en Comunicaciones

${ }^{7}$ Estudiante de la Licenciatura en Negocios Internacionales

${ }^{8}$ Estudiante de la Licenciatura en Administración de Empresas con énfasis en Computación

${ }^{9}$ Estudiante de la Licenciatura en Contaduría Pública
} 
pueblos indígenas posterior a los Acuerdos de Paz, enfocando el accionar de las instituciones de Estado y no gubernamentales en la reivindicación de los derechos humanos de dicha población.

\section{Palabras clave}

Indígenas de El Salvador - aspectos sociales. Derechos humanos - El Salvador. Acuerdos de Paz - El Salvador. Población indígena. El Salvador - problemas raciales. Problemas sociales. Identidad cultural - El Salvador. Violación de los derechos humanos.

\section{Conceptualizando etnicidad, raza, racismo y discriminación}

En el concepto de etnicidad se integra elementos comunes, tales como el idioma, la religión, la tribu, la nacionalidad o la raza, o una combinación de estos, que comparten un sentimiento común de identidad con otros miembros del grupo. Giddens (2000) nos cita a continuacion el concepto de etnicidad:

La etnicidad hace referencia a las prácticas culturales y perspectivas que distinguen a una determinada comunidad de personas. Los miembros de los grupos étnicos se ven a sí mismos como culturalmente diferentes de otros grupos sociales, y son percibidos por los demás de igual manera. Hay diversas características que pueden servir para distinguir a unos grupos étnicos de otros, pero las más habituales son la lengua, la historia o la ascendencia (real o imaginada), la religión y las formas de vestirse o adornarse (p. 2).

Asimismo, es vital analizar los conceptos de raza y racismo para ubicar al lector y a la población en general sobre cómo dichas acepciones han permeado con la violencia psicológica en la población indígena, dando lugar a la negación de sí mismos en las nuevas generaciones, entendiéndose como raza los diferentes tonos de piel, color de ojos, etc. Giddens (2000) cita a continuación dichos conceptos: "Raza en un sentido estricto, no existen las 'razas', sólo variantes físicas of this article is that of studying the phenomenon of social violence towards the indigenous peoples after the Peace Accords; it focuses on the actions taken by State and non-governmental institutions in the recognition of the human rights of said population.

\section{Keywords}

El Salvador Indigenous Peoples-social aspects. Human rights-El Salvador. Peace Accords-EI Salvador. Indigenous Population. El Salvador-racial issues. Social issues. Cultural identity-El Salvador. Human Rights Violations.

en los seres humanos" (p. 4); y "Racismo es un prejuicio que se basa en distinciones físicas socialmente significativas, racista es aquel que cree que ciertos individuos son superiores o inferiores a otros en virtud de estas diferencias raciales" (p.5).

En lo anterior converge de manera apropiada el concepto de discriminación, el cual define a una persona o pueblo, en donde la otredad estudiada por la ciencia antropológica expone una infinidad de contextos socioculturales. Pero antes de entrar en dicho debate, se presenta a continuación el concepto de discriminación acuñado por Giddens (2000), en el que se sitúan los siguientes elementos: "La discriminación es la conducta real que se tiene con un grupo ajeno y puede apreciarse en actividades que le privan de las oportunidades que otros disfrutan, como cuando se le niega a un negro británico el trabajo que se ofrece a un blanco" (p. 5).

\section{La identidad en los pueblos indígenas}

Es difícil en El Salvador actual, distinguir una persona indígena, ya que tradicionalmente se tiene un concepto del indígena por su vestimenta y no por su identidad cultural. CCNIS \& Concultura (1999) nos cita:

El vestido típico ya no existe. Como afirma Mac Chapin, "con excepción de las cofradías, las ceremonias religiosas asociadas y la elaboración de algunas artesanías, los/las indígenas tienen poco 
que los/las distinga del resto de la población. Los pueblos indígenas, en nuestro país, no muestran las características culturales externas y observables de otros pueblos y culturas, como su traje e idioma. En este territorio, los indígenas están "invisibles", incorpóreos, como fantasmas. La mayoría de los residentes de San Salvador afirman que los indios ya no existen, y a los extranjeros se les dice siempre que la cultura indígena se ha abandonado, con excepción, quizás, de algunos núcleos aislados sumamente pobres e insignificantes del sector rural (p. 21).

En el fragmento anterior, se puede observar cómo el fenotipo en una persona no determina necesariamente su etnicidad; es en este punto donde el comportamiento del ser humano define su cultura y su entorno social.

\section{Metodología de la investigación}

Tipo de investigación

En el presente estudio investigativo se aplicó el enfoque cualitativo, que constó de las tres fases o etapas siguientes:

- Análisis documental, donde se exploraron diferentes leyes y documentos oficiales, como convenios internacionales relacionados con el tema de investigación.

- Mapeo de actores, en el cual se buscaron instituciones y personas vinculadas con la defensa de los derechos humanos de los pueblos indígenas u originarios, como también se identificaron actores clave para la realización de entrevistas.

- Entrevistas, para las que se diseñó una guía. Una vez identificadas las personas clave, se llevaron a cabo las entrevistas.

¿Qué es un enfoque cualitativo? Según Sampieri Hernández (2003), el enfoque cualitativo es el que "utiliza la recolección y análisis de los datos para afinar las preguntas de investigación o revelar nuevos interrogantes en el proceso de interpretación" (p. 11).

Además de la primera etapa, que consistió en la consulta de fondos documentales, se definió la técnica de la entrevista guiada, del método cualitativo, mediante la elaboración de un guion de preguntas abiertas, el cual es el instrumento de dicha técnica cualitativa. En ese sentido, las preguntas están estrechamente ligadas con los objetivos de investigación, los cuales determinan los interrogantes de todo proceso de investigación.

La aplicación de dicho instrumento permitió desarrollar la segunda etapa, la cual consistió en registrar los puntos de vista y percepciones de representantes de diferentes instituciones tanto del Estado salvadoreño, entre ellas Secultura, ${ }^{10}$ como de organizaciones no gubernamentales, entre ellas informantes clave que son parte de la población indígena salvadoreña, entre ellos un joven estudiante izalqueño que estudia la Licenciatura en Antropología en la Universidad Tecnológica de El Salvador (Utec).

Finalmente, las entrevistas guiadas realizadas fueron procesadas para realizar el análisis interpretativo de los hallazgos de campo, y así poder socializar los resultados obtenidos mediante un informe final de investigación y su respectivo artículo científico.

La discusión de los resultados mediante la investigación cualitativa

La situación de los pueblos indígenas de El Salvador en torno a la violencia social post Acuerdos de Paz

Pese a los esfuerzos que los diferentes gobiernos, municipalidades y organizaciones de la sociedad civil, creadas después de la firma de los Acuerdos de Paz, la percepción de la situación de los pueblos indígenas en El Salvador, de acuerdo con la licenciada Rita de Araujo (2017), jefa del Departamento de Pueblos Indígenas de la Secultura desde 1995, es la siguiente:

Hay que hablar de la situación que han tenido los pueblos indígenas a través del tiempo, ¿verdad? Han sido una población desestimada, discriminada, invisibilizada. Es decir, realmente, si nosotros vemos en general las cuestiones indígenas, los gobiernos no lo han tratado ¿verdad? Es más, lo del 32 fue algo muy significativo porque hubo una gran matanza. Por eso es que los pueblos indígenas mantienen una lucha; porque dicen: "Si este era nuestro territorio; si estas eran nuestras tierras, ¿verdad? Y como

\footnotetext{
${ }^{10}$ Secretaria de Cultura de la Presidencia de la República de El Salvador
} 
ahora no poseemos nada", ¿verdad? Entonces, los han invisibilizado.

Esto da una idea de que desde el aparato gubernamental se tiene claro que hay esfuerzos; pero que las condiciones de la población indígena son de discriminación e invisibilización.

Asimismo, el estudiante Miguel Najo Latin (2017), procedente de Izalco y de origen indígena, quien cursa la Licenciatura en Antropología de la Utec, afirma lo siguiente:

Todavía son invisibilizados; la discriminación, también. Tenés eso de que vos le preguntas a alguien ' $i y$ vos sos indígena?'. Te dirán: "No, yo no. Yo no soy indio". Hay gente que, si te ve hablando en la cofradía, te dice: "Ah, ve, este está hablando con el indio"; porque se dio la concepción en aquel entonces de que el indígena era el ignorante; aquel que no vale nada. Y todavía existe eso.

Si bien nos damos cuenta, es que no nos han educado para aceptar nuestra propia identidad. Porque vos te das cuenta, en el libro de la colección cipotes, te habla de los indígenas, pero solo en la parte de la Conquista.

Siguiendo con los aportes de Najo Latin (2017), se hace referencia a la precariedad de las condiciones de las poblaciones indígenas de Izalco, al mencionar que muchos de ellos, su puerta la aseguran con una pita de su zapato, una cinta de zapato; eso es con lo que amarra su puerta, porque es gente que vive de lo que sus vecinos le dan; gente que a veces ni come. Porque ¿qué es lo que come la gente? Como el mercado en Izalco se cierra a las cinco de la tarde, la gente va buscando la comida que hay en el rincón antes de que pase el camión de la basura. Esta gente, que de su pobreza sigue con sus tradiciones. Pero ¿por qué? Porque es lo único que tiene, lo único que le queda.

El comentario de Najo Latin sirve para reforzar y reflexionar sobre lo expresado por la representante de Secultura y la manifiesta situación de discriminación e invisibilización de los pueblos indígenas, su cultura y sus tradiciones, así como las condiciones de extrema pobreza que sufren estas poblaciones, producto de la falta de sensibilización por parte de la población mestiza.

Para culminar el punto sobre la situación de los indígenas en El Salvador, cabe destacar que aún existen distintos tipos de violencia hacía los pueblos originarios por diversas causas. El gobierno salvadoreño está haciendo esfuerzos para erradicar la violencia que sufren nuestros pueblos originarios.

Entre las organizaciones no gubernamentales y gubernamentales que trabajan por la defensa de los derechos humanos de los pueblos indígenas están las siguientes:

\begin{tabular}{|l|l|}
\hline \multicolumn{1}{|c|}{ No gubernamentales } & \multicolumn{1}{c|}{ Gubernamentales } \\
\hline Asociación de Consejos de Pueblos Originarios de Cuscatán & Consejo Coordinador Nacional Indígena Salvadoreño \\
\hline Los pasos del Jaguar & Secretaria de Cultura. Secultura \\
\hline Asociación de Nacional de Indígenas Salvadoreña & Procuraduría para la Defensa de los Derechos Humanos \\
\hline Consejo de los Pueblos Originarios Nahuat Pipil, Nahizalco \\
\hline Organización Winaka, Cacaopera \\
\hline Asociación Coordinadora de Comunidades Indígenas de El Salvador \\
\hline Instituto para el Rescate Ancestral Indígena Salvadoreño \\
\hline
\end{tabular}

\section{Legislación nacional e internacional}

A partir de la fase I de análisis documental y de la fase II de las entrevistas, se encontraron diferentes leyes del Estado salvadoreño, ordenanzas municipales de Izalco, Nahuizalco, Santo Domingo de Guzmán, Panchimalco, Conchagua y
Cuisnahuat, y convenios internacionales relativos a los pueblos originarios.

Mucha de esta información se logró identificar a partir de la entrevista realizada a la Licda. Rita Jaimes de Araujo (2017), quien destaca las leyes que respaldan los 
derechos de los indígenas a escala nacional; entre ellas están las siguientes:

Ley de la Cultura, Artículo 3, seis ordenanzas municipales que son pequeñas leyes dentro de un municipio; por ejemplo, tienen Izalco, Nahuizalco, Santo Domingo de Guzmán, Panchimalco, Conchagua y Cuisnahuat, que trabajan con las alcaldías,; Ley del
Artesano y muchos instrumentos internacionales que el país ha realizado en relación con los pueblos indígenas.

Las leyes nacionales y los convenios internacionales sistematizadas por el grupo investigador, de acuerdo con los hallazgos en la entrevista realizada, se presentan en el siguiente cuadro.

\section{Cuadro 1. Legislación nacional e internacional}

\begin{tabular}{|l|}
\hline \multicolumn{1}{|c|}{ Legislación nacional } \\
\hline Constitución de la República \\
Ratificación de la incorporación de un inciso al Art. 63 de la \\
Constitución, hecha el 25 de abril de 2012, reconociendo a \\
los pueblos indígenas.
\end{tabular}

Ley de Cultura (2016)

Art. 5.- El Estado debe garantizar el ejercicio de la libertad creativa, la educación artística, la diversidad cultural, los derechos de los pueblos indígenas, el respeto a los derechos y dignidad de los artistas y creadores y a sus obras, y el rescate, protección y enriquecimiento del patrimonio cultural tangible e intangible de la nación.

Ley de Fomento, Protección y Desarrollo del Sector Artesanal con énfasis en los pueblos indígenas (2016) Ar t. 1.- La presente Ley tiene por objeto fomentar, proteger y desarrollar al sector artesanal, reconociendo como pilar fundamental a la persona dedicada a la producción de artesanías, con énfasis a las personas de origen indígena, y las que con su producción generan identidad y enriquecen el patrimonio cultural y el desarrollo del país.

Ordenanza municipal de Izalco, Sonsonate (2012). 34 artículos

Art. 3.- La municipalidad de Izalco reconoce a la comunidad indígena del municipio cuyos miembros descienden de los primeros pobladores de este territorio que hoy se conoce como Izalco.
Convención Internacional para la Eliminación de Todas las Formas de Discriminación Racial. (1969)

La Declaración de los Derechos de los Pueblos Indígenas, de las Naciones Unidas (2007)

Artículo 1

Los indígenas tienen derecho, como pueblos o como individuos, al disfrute pleno de todos los derechos humanos, y las libertades fundamentales, reconocidos en la Carta de las Naciones Unidas, la Declaración Universal de Derechos Humanos y las normas internacionales de derechos humanos.

Convenio 169 sobre pueblos indígenas y tribales. (1989)

El cual solo 22 países lo han ratificado. A escala de Latinoamérica los países que lo han ratificado son: Argentina, Bolivia, Brasil, Chile, Colombia, Costa Rica, Ecuador, Guatemala, Honduras, México, Nicaragua, Paraguay, Perú y Venezuela.

No lo han ratificado 165 países, incluido El Salvador.

Convenio Constitutivo del Fondo para el Desarrollo de los Pueblos Indígenas de América Latina y el Caribe. (1992) Aprobado en la II Cumbre Iberoamericana para apoyar los procesos de autodesarrollo de pueblos, comunidades y organizaciones indígenas. 
Ordenanza municipal de Nahuizalco, Sonsonate (2010). 38 artículos

Art. 3.- La Municipalidad de Nahuizalco reconoce a los pueblos y comunidades indígenas del municipio quienes se asentaron hace varios, miles de años y por tanto son los primeros pobladores de este territorio que hoy se conoce como municipio de Nahuizalco.
Ratificación del convenio 107. Convenio sobre poblaciones indígenas y tribuales. (1957)

El cual solo 27 países lo han ratificado. A escala de Latinoamérica, los países que lo han ratificado son: Argentina, Bolivia, Brasil, Colombia, Costa Rica, Cuba, República Dominicana, Ecuador, México, Panamá, Paraguay, Perú y El Salvador.

Ordenanzas municipales entorno a la etnicidad de la población indígena salvadoreña

Ordenanza municipal de Santo Domingo de Guzmán, Sonsonate

No se cuenta con el texto de la ordenanza.

Ordenanza municipal de Pachimalco, San Salvador (2015)

Art. 1.- La Municipalidad de Panchimalco reconoce a la comunidad indígenas del municipio de Panchimalco, cuyos miembros mujeres y hombres, descienden de los primeros pobladores de este territorio que hoy se conoce como Panchimalco.

Ordenanza municipal de Conchagua, La Unión

No se cuenta con el texto de la ordenanza.

\section{Ordenanza municipal de Cuisnahuat, Sonsonate (2015)}

Art. 3.- La municipalidad de Cuisnahuat reconoce a la comunidad indígena del municipio cuyos miembros descienden de los primeros pobladores de este territorio que hoy se conoce como Cuisnahuat.

(Elaboración propia)

Además, se encontraron otros instrumentos de política pública de El Salvador, de las cuales resaltan la Política Nacional de Pueblos Indígenas, pendiente de su lanzamiento oficial, iniciada su elaboración desde el 2015, pero no oficializada, y el Plan de Acción Nacional de los Pueblos Indígenas, el cual inició su elaboración en 2015 y finalizó en el 2017.

No existe una ley específica o especial que vele por los derechos de la población indígena en El Salvador, pese a estar reconocida en la Constitución de la República. Son particularmente interesantes las ordenanzas que han surgido desde el 2010 y que van reconociendo a los pueblos indígenas en dichos municipios.

Sin embargo, la mera existencia de leyes u ordenanzas no garantiza el goce de derechos. De acuerdo con Najo Latin (2017), la efectividad de las leyes nacionales está por debajo de lo deseado. $Y$ es que las leyes habilitadas no tienen el impacto que se espera para la inclusión y la defensa de los derechos de la población indígena; y afirma lo siguiente:

Pues hasta el momento no han habilitado nada; porque, vaya, creo que esa notificación ya tiene bastante, desde 2010, si mal lo recuerdo, por ahí anda, no recuerdo la fecha. Pero ¿qué pasa, por ejemplo, en el caso de Izalco? Cuando vos llegás y les preguntás ¿qué ha hecho hasta ahorita el Gobierno? Hasta el momento nada, o sea, las personas te dicen: "Pues sí. Pero es que a nosotros nos utilizan". Y sí, eso es verdad; porque ¿en qué los utilizan? En este aspecto, mirá, vuelvo y te repito: anteriormente este tema no era muy sonado. Entonces ¿qué pasa? Hay gente que siempre intenta aprovecharse de eso.

Se necesita una mayor voluntad política, por parte del gobierno central y de los partidos representados en la Asamblea Legislativa, para aprobar instrumentos nacionales o internacionales, como el Convenio 169 de la Organización Internacional del Trabajo (OIT), para que amparen a las poblaciones indígenas de El Salvador.

Organizaciones que trabajan por la defensa de los derechos humanos de los pueblos originarios de El Salvador

Existen diversas organizaciones, algunas gubernamentales y otras no gubernamentales, que trabajan para la defensa de los pueblos originarios de El Salvador. 
Entre las organizaciones gubernamentales está la Secretaría de la Cultura de la Presidencia de la República de El Salvador (2017), la cual se encarga de "velar por la conservación, fomento y difusión de la cultura, proponiendo políticas culturales y planificando, organizando y dirigiendo las diversas formas de investigación, formación artística, apoyo a la creación popular, salvaguarda, restauración y difusión del patrimonio cultural del país".

Asimismo, Secultura ${ }^{11}$ es el ente rector de la Ley de Cultura aprobada por la Asamblea Legislativa (2016), la cual busca la promoción de las diferentes expresiones culturales, incluidas las de la población indígena, como se menciona en el artículo 5: "El Estado debe garantizar el ejercicio de la libertad creativa, la educación artística, la diversidad cultural, los derechos de los pueblos indígenas, el respeto a los derechos y dignidad de los artistas y creadores y a sus obras, y el rescate, protección y enriquecimiento del patrimonio cultural tangible e intangible de la nación".

La valoración de la Licda. Rita Jaimes de Araujo (2017), al ser consultada sobre las organizaciones que trabajan por la defensa de los derechos humanos de los pueblos originarios de El Salvador, fue la siguiente:

En los Acuerdos de Paz no se menciona a los pueblos indígenas, pero sí se reconocen como un surgimiento de las organizaciones indígenas, que venían ya desde un movimiento internacional, promoviendo el derecho de reconocimiento de su cultura hacia esos pueblos. La primera organización indígena empieza desde los 60.

La primera organización es la Asociación Nacional de Indígenas Salvadoreños (Anis); el cacique era don Adrián Espino Lisco. Esta organización se va desmembrando, Cuando comencé a trabajar en el Departamento de Pueblos Indígenas, en el año1995, había alrededor de 25 organizaciones. Ahora existen muchas más.

Cuando nace Anis, hay cierta cultura; pero se ha ido perdiendo. Por ejemplo, el idioma. Realmente el Estado o el Ministerio de Educación no han hecho nada para dar a conocer, a todos los salvadoreños, el tema de los pueblos indígenas. Solo se habla (de ellos) por ejemplo el 12 de octubre, pero no se habla de esa cultura indígena que halló Colón. Se habla de los viajes de Colón; de que trajo las tres carabelas.

En el año 1958 nace la Organización Internacional del Trabajo; hace un convenio, el 107, donde habla de la población indígena, que dice este convenio que es una simulación; que estos pueblos tienen que ser asumidos por la cultura de los mayores; de los que mandan, o sea, los del poder. Luego surge el convenio 169, que ya dice que son pueblos que deben ser reconocidos.

Se demuestra que hay conocimiento, por parte de las instituciones del gobierno central, del desarrollo histórico de las organizaciones y de los diferentes tipos de vulneraciones que se hacen hacia los pueblos indígenas de El Salvador.

\section{Fotografía 1}

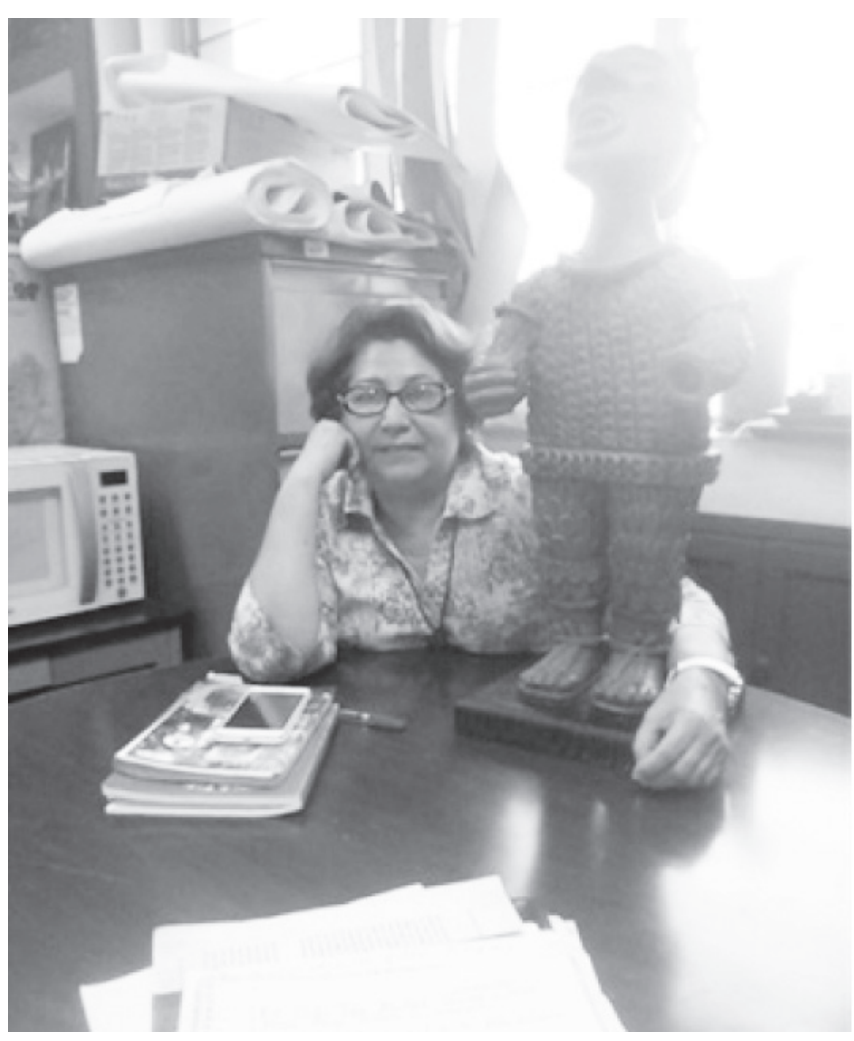

Entrevista con la Licda. Rita de Araujo, jefa del Departamento de Pueblos Indígenas de la Secultura. Fotografía tomada por equipo de investigación.

\footnotetext{
${ }^{11}$ Abreviatura de Secretaria de Cultura de la Presidencia de El Salvador.
} 


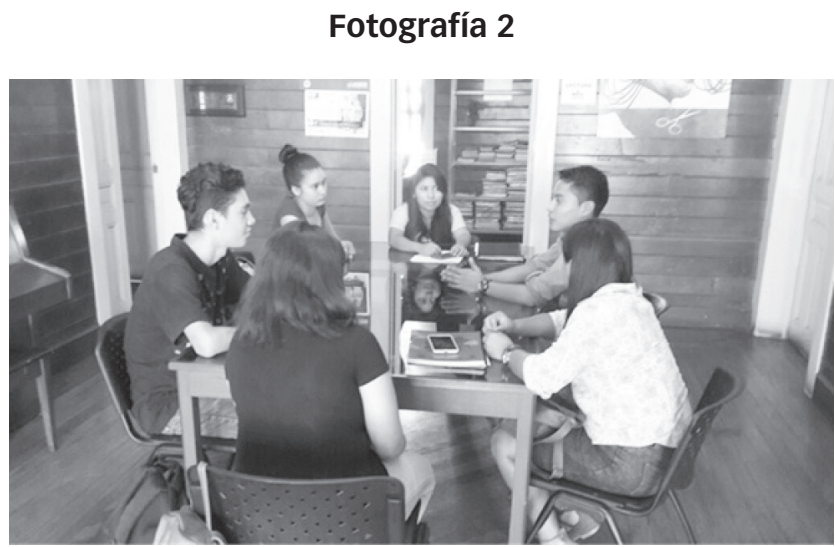

Momento de la entrevista guiada con Miguel Najo Latin, estudiante de Antropología de la Utec. Fotografía tomada por equipo de investigación.

Desde el punto de vista de Najo Latin (2017), como indígena, en referencia a cuáles son las organizaciones que trabajan por la defensa de los derechos humanos de los pueblos originarios de El Salvador, tal como se expresó en el siguiente aporte:

Está la organización Feliciano Ama; está ubicada en Izalco, formada en la unidad de Feliciano Ama. Y es curioso, porque ella se hace llamar perteneciente a ese linaje Ama; pero cuando vos ves a la propia familia de Feliciano Ama, no está metida en esas cosas.

La otra sí no sé de dónde es, pero se llama la Red de Jóvenes Indígenas de El Salvador, que obviamente son jóvenes; se sientan a platicar sobre el tema. Pero, que yo sepa, que están desarrollando un programa para la población indígena, no; porque es lo que les digo, se limitan más a dar a conocer que nosotros somos indígenas, a exigir cosas que quizás la pobre gente no da. Es como esa fundación Feliciano Ama. Ahorita están velando para que se les den tierras. Pero ¿quiénes creés que van a ser los beneficiados? Vos pensás que la gente indígena. No. Imagínate que ellos (tienen) casas de dos, tres plantas. Entonces, es bien indignante el hecho de que tienes a gente (organizaciones) que se están lucrando de ellos y que con ese dinero construye su casa y mejora sus carros; y tenés a gente indígena que no le alcanza para vivir.

Es importante que existan diferentes organizaciones que defiendan los derechos de los pueblos originarios, pero se requiere que estas organizaciones trabajen con transparen- cia y verdaderamente por los pueblos originarios, por intereses colectivos y no personales.

Desafíos que enfrentan las diferentes organizaciones que trabajan para la defensa de los derechos humanos de la población indígena salvadoreña

Como se puede empezar a vislumbrar, cuando se tienen en cuenta la falta de legislación, las condiciones de discriminación, de pobreza y la forma como operan las organizaciones no es difícil prever que estas enfrentan diversos desafíos.

Como menciona Najo Latín (2017) sobre los desafios de la población indígena en la actual sociedad salvadoreña en el siguiente comentario:

Mira una cosa lleva a la otra tenemos el problema de que la gente se aprovecha de ellos, por eso es que entrar a una cofradía o a un cierto grupo es bien complicado, esa misma discriminación que te violenta tus derechos hace que este grupo se cierre y te es difícil entrar porque yo tengo un caso de un amigo bueno puedo poner el ejemplo de mi mamá, fíjate que mi mamá pertenece a una iglesia de por ahí y pasó como tres años pidiendo un proyecto para darles comida y todo eso y no pudo entrar y pasaron insistiendo e insistiendo hasta tres años después que los dejaron entrar.

También, que hay un caso de un mayordomo de una cofradía; de que fue así: él le enseñó lo que sabía; le enseñó sobre las cofradías a un muchacho, y este muchacho era hijo de una persona que pertenece a una organización. Y viene y le enseña; y gracias a lo que él aprendió gana una beca para Italia. Después de Italia pasa a Arabia, como por esa zona. Después viene el muchacho, regresó, y le traía su cajita de tierra; era arena. Bueno. Entonces el muchacho regresa con la mamá, que era líder de esa organización. Y, bueno, llega el muchacho y le dice: "Buenas. Mire, aquí le traigo un recuerdo". Y se lo agarra. Y viene una mujer, y le dice: "Él le está dando eso, pero no crea que eso fue lo que el gano. Él no se ganó esa beca por lo que usted le enseñó". O sea, imagínate, gracias a él gana la beca; él le lleva solamente arena y encima le dice que no fue gracias a él. O sea, por eso es que hay gente indígena que no tiene más confianza con las demás personas. Aparte de esto, tienes el problema de la pobreza. 
Para ayudar a los indígenas se tiene que comenzar desde cero, porque, como el problema que hay en el país es que como no nos inculcaron respeto a eso, tenés que inculcar desde el niño; tiene que ver desde la educación, desde las leyes y desde las alcaldías, que sería el ente de estas personas incluirlas, conservar sus tradiciones. $Y$ entra otro factor, que es el turismo.

Lastimosamente, ese es el problema se sigue utilizando a la población indígena para ganar fama, para ganar dinero; y no se les ayuda en nada.

Uno de los desafíos que se puede observar es el abuso de parte de las personas mestizas hacia los indígenas, al aprovecharse de sus conocimientos ancestrales para beneficios personales. Además, la falta de solidaridad entre los mismos indígenas pertenecientes a las diferentes organizaciones, quienes, de acuerdo con lo que manifiesta Najo Latin (2017), solo velan por los intereses de quienes pertenecen a sus organizaciones y no de los demás indígenas.

\section{Conclusiones}

- Mediante el presente trabajo de investigación, se ha explorado la situación de la violencia social post Acuerdos de Paz que sufre la población indígena de El Salvador. Esta violencia se traduce en discriminación, ya que en la práctica los indígenas no gozan de los mismos derechos que los demás salvadoreños ni de sus derechos como pueblos originarios, tales como la protección de sus lenguas, su cultura y costumbres. Los pueblos indígenas también son invisibilizados, ya que no se tomen en cuenta a la hora de formular políticas públicas; se les trata como un colectivo homogéneo, cuando no es así pues forman parte de diferentes pueblos, como los nahua pipiles, los chortis, los lencas, los kakawira, entre otros. Finalmente, y por si fuera poco, sufren violencia, ya que son utilizados para que otras organizaciones no indígenas logren proyectos que no los benefician.

- Se conocen artículos de la Constitución, algunas leyes nacionales, ordenanzas municipales y convenios internacionales ratificados que favorecen los derechos humanos de los pueblos originarios de El Salvador. Sin embargo, falta una ley específica que ampare la totalidad de los derechos humanos de esta población indígena. Y, pese a que existe legislación, esta no protege de manera efectiva a la población indígena, ya que sufre de discriminación, invisibilización y utilización de parte de la población no indígena.

- Existen organizaciones que trabajan por la defensa de los derechos humanos de los pueblos originarios, tanto del Gobierno como no gubernamentales. Pero, aunque existen estas organizaciones, no siempre trabajan por los intereses de la totalidad de los pueblos originarios, sino que, en algunos casos, solo por intereses personales. Las organizaciones indígenas se enfrentan a los desafíos de aumentar su número de miembros, desarrollar proyectos inclusivos que beneficien al mayor número de sus miembros, y sobre todo tienen el reto y el deber de sensibilizar a la población mestiza acerca de la importancia de los pueblos originarios en el rescate y la construcción de la identidad.

\section{Referencias}

Araujo, R. de. (2017). Estudios de los pueblos indígenas y la violencia social post acuerdos de paz en El Salvador. El Salvador.

Asamblea Legislativa. (2016). Ley de cultura. Recuerado de http://www.cultura.gob.sv/ley-de-cultura/

Asamblea Legislativa. El Salvador. (2014). Constitución de la República de El Salvador. El Salvador. Recuperado de http://www.pgr.gob.sv/documentos/ConstitucionDe LaRepublica.pdf

Consejo Coordinador Nacional Indigena Salvadoreño. Organización Panamericana de la Salud. El Salvador: CCNIS - CONCULTURA. \& OPS/OMS EL SALVADOR. (1999). Pueblos indigenas salud y calidad de vida en El Salvador. Recuperado de http://www.bvsde.paho.org/ tutorialin/pdf/pueblos_indigenas.pdf

Giddens, A. (2000). Etnicidad y Raza. Madid: Alianza. Recuperado de https://centroderecursos.cultura.pe/ sites/default/files/rb/pdf/Etnicidad\%20y\%20raza.pdf

Hernández Sampieri, R., Fernández Collado, C., \& Baptista Lucio, P. (2014). Metodología de la investigación (6 ${ }^{\mathrm{a}}$ ed.). México, D.F.: McGraw-Hill/ Interamericana.

Iniciativa Social para la Democracia. (2015). Derechos de los pueblos indígenas. Recuperado de http://www.isd. org.sv/index.php/comunicacion-para-la-democracia/ derechos-de-los-pueblos-indigenas 
Latín, M. N. (Noviembre de 2017). Estudios de los pueblos indígenas y la violencia social post acuerdos de paz en El Salvador. (W. Mónico, Entrevistador)

Naciones Unidas. (2015). Declaracion Universal de los Derechos Humanos. Recuperado de http://www. un.org/es/documents/udhr/UDHR_booklet_SP_ web.pdf
Naciones Unidas, D. H. (2013). Los pueblos indígenas y el sistema de derechos humanos de las Naciones Unidas. Recuperado de http://www.ohchr.org/Documents/ Publications/fs9Rev.2_sP.pdf

Secretaría de la Cultura de la Presidencia de la República de El Salvador. (2017). Secretaria de Cultura. Recuperado de http://www.cultura.gob.sv/secretario-de-cultura-2/ 